\title{
Using Device Data to Improve Identification of Intraoperative Femur Fractures in Total Hip Arthroplasty
}

Author Affiliations: 'University of Michigan Medical School; ${ }^{2}$ Michigan Arthroplasty Registry Collaborative Quality Initiative (MARCQI), Ann Arbor, MI, 48109, USA; ${ }^{3}$ Department of Orthopaedic Surgery, University of Michigan, Ann Arbor, MI, 48109, USA; ${ }^{4}$ Quality Institute, St Joseph Mercy Hospital, Ann Arbor, Ml, 48109, USA; Department of Orthopaedic Surgery, Sparrow Health System, Lansing, MI 48912, USA

\section{Author E-mail List:}

aaomari@med.umich.edu,

rehughes@med.umich.edu,

hallstro@med.umich.edu,bsingal@med .umich.edu, igrisanr@med.umich.edu, brian@mccardel.us

Corresponding Author: Richard E.

Hughes, PhD (rehughes@umich.edu)

Disclaimer: Although Blue Cross

Blue Shield of Michigan (BCBSM) and the MARCQI work collaboratively, the opinions, beliefs, and viewpoints expressed by the authors do not necessarily reflect the opinions, beliefs, and viewpoints of BCBSM or any of its employees.

Conflicts of Interest: The

authors have no conflicts

of interest to disclose.

Amro Omari, $\mathrm{BS}^{1}$; Richard E. Hughes, $\mathrm{PhD}^{2,3}$; Brian R. Hallstrom, MD ${ }^{2,3}$; Bonita M. Singal, MD, PhD ${ }^{2,4}$; Rochelle M. Igrisan, $\mathrm{RN}^{2,3}$; and Brian R. McCardel, $\mathrm{MD}^{2,5}$

\section{Introduction}

I

ncreased population longevity and advances in health care technologies have boosted the need for joint replacement operations. Approximately 2.5 million Americans are living with a Total Hip Arthroplasty (THA), with the yearly surgical prevalence being $0.82 \%$ of the population. ${ }^{1}$ One of the most feared operative complications of THAs is intraoperative femoral fractures. Studies show intraoperative fractures occur in approximately $0.1 \%$ to $1 \%$ of cemented THAs and 3\% to $18 \%$ of uncemented THAs. ${ }^{2}$ Given the potential consequences, increased emphasis needs to be placed on identifying intraoperative fractures during THAs and on reducing the rates of fracture complications that occur during joint replacements.

Surgical reports filled out perioperatively by the surgeon can be inspected manually to identify intraoperative femoral fractures. ${ }^{3-10}$ However, the ambiguity in the wording of surgical reports can be a source of error in this process, and note of an intraoperative fracture can be missed. Cerclage cables are devices used in primary THAs for the prevention of repair of fracture. If the surgeon is concerned that a fracture is likely due to poor bone quality or other factors, then a prophylactic cable is placed prior to broaching or stem insertion. In this study, we investigated the utility of data abstraction from orthopedic registries as a more accurate method of capturing intraoperative femoral fractures via identification of the use of cerclage cables.

\section{Methods}

This study was conducted using the Michigan Arthroplasty Registry 
Collaborative Quality Initiative (MARCQI), which is a statewide consortium of hospitals and providers that has an online database containing information about the arthroplasty surgeries performed and the devices implanted for each orthopedic operation in MARCQI participating hospitals. ${ }^{11}$ The analysis included all THA cases registered in MARCQI from April 1, 2012, to December 31, 2013. For each case, the following variables were extracted from the database: (1) case number, (2) surgeon ID, (3) hospital ID, (4) whether a cerclage cable was used in the case, (5) date of surgery, and (6) whether an intraoperative femoral fracture was reported as a complication.

THAs in which cerclage cables were used were identified. Of those cases, if no intraoperative femoral fracture was reported, case number and date of surgery were provided to a data abstractor at each hospital so they could rereview the medical record for evidence of an intraoperative fracture (Figure 1). For each case they reviewed, abstractors were asked to extract the following data: (1) if cerclage cable use was recorded in the operative note,
(2) if a fracture was suspected, (3) location of fracture, (4) if the cable was used prior to broaching, (5) and if the planned femoral stem was changed during the case. Abstractors were instructed to look for the words crack, split, opening, fragment, and restricted weight bearing postoperatively because surgeons sometimes use words other than "fracture" to indicate a fracture in their dictations of the operation. In addition, free text fields were provided for abstractor notes, and text was copied directly from the medical record.

From the extracted data, an orthopedic surgeon determined whether an intraoperative fracture had occurred. Intraoperative femoral fractures were excluded if the operating surgeons explicitly noted that the cables were used for prophylaxis or before broaching, if a fracture occurred in the pelvis rather than the femur, or if there was no objective evidence for an intraoperative fracture recorded in the medical record. Descriptive statistics were computed using SAS 9.4 (SAS Institute, Cary, NC). Fischer's exact analysis was performed to look for statistically significant associations

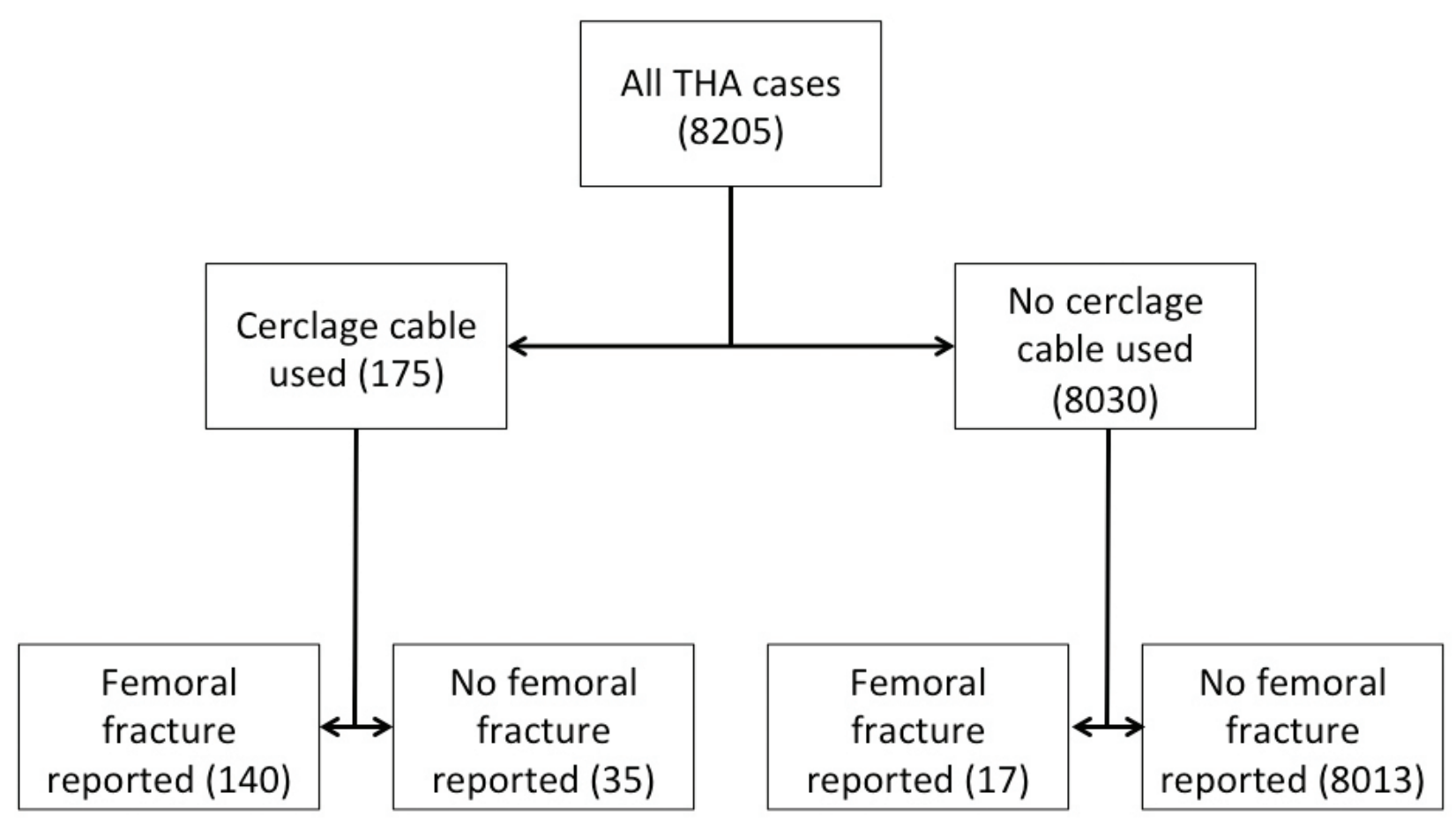

FIGURE 1. Study population flow chart 
between the purchase of a cerclage cable and an intraoperative femoral fracture.

\section{Results}

Between April 1, 2012, and December 31, 2013, 8205 primary THAs were identified. Of the 8205 THAs, 184 (2.24\%) cases reported use of a cerclage cable. Prior to case rereview, out of the 8205 cases, there were 115 (1.40\%) cases in which intraoperative femoral fractures were reported. Cerclage cables were used in 83 of these cases. This left 101 cases in which cerclage cable use was reported, but no fracture was reported. When these 101 cases were rereviewed, via the methods described above, an additional 42 fractures were identified. This secondary review brought the total number of intraoperative fractures up from 115 to 157, leading to a revised risk estimate of intraoperative femoral fracture during primary THA of 1.91\% [Table 1]. Fischer's exact test for cerclage cable presence and fracture occurrence yielded significant association $(p<0.0001)$.

\section{Discussion}

Our results demonstrate that registries can leverage device data to capture complications such as femoral fractures that would otherwise be missed when relying on self-reporting in operative reports or in administrative data. This provides abstractors with a tool to find fractures when the wording in the surgical report is ambiguous. One clinical implication of this finding is for hospital quality improvement efforts. Quality improvement activities in arthroplasty need to properly capture complications, and these results show that such efforts should include review of device data for the use of cables. A more accurate capture of fractures will be important as the relationship among early failure, stem design, fixation technique, and survivorship is explored. ${ }^{13-15}$ This can potentially save the health care system millions of dollars through reducing the number of revision surgeries due to fractures.

Our study has several limitations. First, not all 8205 cases were rereviewed with the standardized methodology described above. A rereview of all cases may have had an effect on the reported incidence of the intraoperative femoral fractures. Second, although rereview was thorough and done by an expert, the process was still manual, and thus human error was possible. In addition, we can only make conclusions about the use of cerclage cables, but not other fixation methods. For example, we did not account for the purchase of wires that can be used to treat or prevent these fractures because wires are not accounted for with individual bar codes or billing codes in implant data. Follow-up studies should incorporate a more thorough rereview by multiple experts to reduce human error and subjectivity.

Additional studies are required to determine whether device data can be leveraged to improve the capture of complications in other orthopedic surgeries. For example, one such study could include the use of stems to capture complications in primary total knee arthroplasty. The use of a femoral stem may mean a complication has occurred, such as femoral notching, and

TABLE 1. Intraoperative Femur Fractures and Cerclage Cables Found After Rereview of Cases Containing Cerclage Cables

No Intraoperative Fracture

8013
Fracture Identified
35

Cable used

Total number of fractures
140

$157(1.91 \%)$ 
that the stem was used to transfer the load for fixation.

\section{Conclusion}

Identifying cases using cerclage cables can help improve the capture of intraoperative femoral fracture in THA cases during data abstraction. This is important to guide collaborative efforts to reduce the clinical and financial burden of this complication. Furthermore, this study provides a methodology behind using orthopedic registries as a potential tool for finding complications when going through ambiguous surgical reports, which can be applied to other specialties.

\section{Acknowledgments}

Support for the Michigan Arthroplasty Registry Collaborative Quality Initiative is provided by Blue Cross and Blue Shield of Michigan (BCBSM) and Blue Care Network as part of the BCBSM Value Partnerships program.

\section{References}

1. Maradit Kremers $H$, et al. Prevalence of total hip and knee replacement in the United States. J Bone Joint Surg Am. 2015;97(17):1386-1397.

2. Saleh $\mathrm{KJ}$, et al. Complications of total hip arthroplasty. Am J Orthop. 2002;31(8):485-488.

3. Gioe TJ, et al. Implementation and application of a community total joint registry: a twelve-year history. J Bone Joint Surg Am. 2006;88(6):1399-1404.

4. Berry DJ, Kessler M, Morrey BF. Maintaining a hip registry for 25 years. Mayo Clinic experience. Clin Ortho Relat Res. 1997;(344):61-68.

5. Carothers JT, et al. Lessons learned from managing a prospective, private practice joint replacement registry: a 25-year experience. Clin Orthop Relat Res. 2013;471(2):537-543.
6. Briant $\mathrm{R}$, et al. Representative case series from public hospital admissions 1998 II: surgical adverse events. N Z Med J. 2005;118(1219):U1591.

7. Espehaug $B$, et al. Early revision among 12,179 hip prostheses. A comparison of 10 different brands reported to the Norwegian Arthroplasty Register, 1987-1993. Acta Orthop Scand. 1995;66(6):487-493.

8. Graves SE, et al. The Australian Orthopaedic Association National Joint Replacement Registry. Med J Aust. 2004;180(5 Suppl):S31-S34.

9. Prime MS, Palmer J, Khan WS. The National Joint Registry of England and Wales. Orthop. 2011;34(2):107-110.

10. Paxton EW, et al. Comparison of the Norwegian knee arthroplasty register and a United States arthroplasty registry. J Bone Joint Surg Am. 2011;93(Suppl 3):20-30.

11. Hughes RE, et al. Michigan Arthroplasty Registry Collaborative Quality Initiative (MARCQI) as a model for regional registries in the United States. Orthop Res Rev. 2015;7:47-56.

12. Hailer NP, et al. The risk of revision due to dislocation after total hip arthroplasty depends on surgical approach, femoral head size, sex, and primary diagnosis: an analysis of 78,098 operations in the Swedish Hip Arthroplasty Register. Acta Orthop. 2012;83(5):442-448.

13. Hailer NP, Garellick G, Karrholm J. Uncemented and cemented primary total hip arthroplasty in the Swedish Hip Arthroplasty Register. Acta Orthop. 2010;81(1):34-41.

14. Taunton MJ, et al. Early postoperative femur fracture after uncemented collarless primary total hip arthroplasty: Characterization and results of treatment. J Arthroplasty. 2015;30(11):2008-2011.

15. Watts CD, et al. Increased risk of periprosthetic femur fractures associated with a unique cementless stem design. Clin Orthop Relat Res. 2015;473(6):2045-2053. 